Rev. Int. Contam. Ambie. 37, 389-400, 2021

https://doi.org/10.20937/RICA.53450

\title{
ANÁLISIS DE OPINIÓN DEL AUTOTRANSPORTE DE PASAJEROS EN MÉXICO, USO DE ENERGÍAS ALTERNATIVAS Y DISMINUCIÓN DE $\mathrm{CO}_{2}$
}

\author{
Analysis of opinion of passenger transportation in Mexico, the use of alternative energies and decrease of $\mathrm{CO}_{2}$
}

\author{
Rosalina BÁEZ MARTÍNEZ
}

Universidad Anáhuac México, Campus Norte, Av. Universidad Anáhuac 46, Col. Lomas Anáhuac, 52786 Huixquilucan, Estado de México, México.

Correo electrónico: rosybaezmartinez@yahoo.com.mx

(Recibido: noviembre 2018; aceptado: noviembre 2019)

Palabras clave: estrategia sostenible, energía fósil, energías limpias, ambiente.

\section{RESUMEN}

El objetivo de la presente investigación fue dar a conocer las preferencias de las empresas de autotransporte federal de pasajeros en México con relación a diferentes tipos de energías alternativas, ya que hasta hoy no han dejado de depender de la energía fósil (principalmente diésel). Esto, tomando en cuenta que la migración hacia el uso de energías alternativas puede representar una estrategia sostenible que ayude a mitigar el daño al ambiente debido a altas emisiones de $\mathrm{CO}_{2}$. El instrumento utilizado fue una encuesta en escala Likert, con 13 preguntas y cinco valores de respuesta: 5) totalmente de acuerdo, 4) de acuerdo, 3) ni de acuerdo ni en desacuerdo, 2) en desacuerdo, 1) totalmente en desacuerdo. Los resultados arrojaron que los empresarios están dispuestos a adoptar una estrategia de sostenibilidad que disminuya el daño al ambiente causado por el uso de energía fósil.

Key words: sustainable strategy, fossil energy, clean energy, environment.

\begin{abstract}
The objective of the present study was to show the preferences of the federal passenger transportation companies in Mexico regarding the different types of alternative energies, since until today they still depend on fossil energy (mainly diesel). Taking into account that migrating towards the use of alternative energies can represent a sustainable strategy that helps to mitigate environmental damage due to high $\mathrm{CO}_{2}$ emissions. The instrument used was a Likert scale survey, with 13 questions and five response values: (5) strongly agree, (4) agree, (3) neither agree nor disagree, (2) disagree, (1) strongly disagree. The results showed that entrepreneurs are willing to adopt a sustainability strategy that reduces damage to the environment caused by the use of fossil energy.
\end{abstract}




\section{INTRODUCCIÓN}

El cambio climático es considerado como una amenaza para las condiciones de vida humana en el planeta. El calentamiento global está provocando variaciones de temperatura y precipitación en todas las regiones de la Tierra; esto incide con efectos directos e indirectos, visibles y no tan visibles, sobre las condiciones de vida de la población en el mundo (Corona 2018). El cambio climático está acompañado de un deterioro ambiental y el transporte terrestre es considerado como una de las principales causas de los impactos negativos que se producen en el ambiente (Lara et al. 2009).

Cabe destacar que la importancia de este transporte radica en que mueve al $96 \%$ de las personas en la República Mexicana de acuerdo con la Estadística Básica del Autotransporte Federal (SCT 2017), por lo cual es fundamental dar a conocer qué tan de acuerdo están las empresas operadoras en buscar mecanismos para mitigar el daño al ambiente; como el establecimiento de políticas empresariales y uso de nuevas tecnologías que reduzcan la emisión de gases efecto invernadero (principalmente $\mathrm{CO}_{2}$ ). Se hace referencia a las políticas, tecnologías y medidas tendientes a limitar y reducir las emisiones de gases de efecto invernadero (principalmente $\mathrm{CO}_{2}$ ) originados por este tipo de transporte, a fin de disminuir su impacto y lograr el decrecimiento de la contaminación ambiental.

La Comisión Mundial del Medio Ambiente y el Desarrollo (CMMAD 1987) menciona que dentro de los esfuerzos iniciales para concientizar al mundo sobre los daños al ambiente de origen antrópico, se encuentra en el Informe Brundtland (CMMAD 1987), en el que se habla de un primer intento para eliminar la confrontación entre desarrollo y sostenibilidad. Se trata de un análisis de la situación mundial a fines del siglo XX y su incidencia en el entorno global, el cual subraya la necesidad de revertir la degradación actual a partir de la consideración del ambiente y del desarrollo como un todo inseparable. De este documento se desprende el concepto vigente de desarrollo sostenible, aplicable a las organizaciones empresariales contemporáneas, el cual fue incorporado posteriormente a todos los programas de la Organización de las Naciones Unidas (ONU) en torno al tema. Por ello fue necesario buscar estrategias desde las empresas para paliar los efectos negativos sobre nuestro planeta. Al respecto, Minzberg y Quinn (1988) mencionan que serán alcanzados los objetivos empresariales para un entorno sostenible a través de la alta dirección de la empresa, desde la que debe partir toda iniciativa. Asimismo, Thompson et al. (2012) indican que las empresas tienen una responsabilidad social corporativa elemental, debido a su compromiso inexcusable con el entorno. Por su parte, Chan y Maubourne (2005) señalan que un desafío para las compañías es crear espacios seguros en el mercado en los cuales la competencia no tenga importancia (océano azul), un plan de proyección como en la "planeación prospectiva, donde es posible concebir futuros alternativos" (Miklos y Tello 1998). Lo anterior, aunado a un cambio hacia la sostenibilidad que represente el interés de los encargados de la toma de decisiones, así como una renovación de procesos y productos a través de una verdadera innovación, que sea una estrategia dirigida al cuidado ambiental para que le agreguen valor a sus empresas como "ventaja competitiva" (Porter 1999).

Según Trentadue y Carranza (2014) el modo terrestre carretero, representa el $79 \%$ del consumo total de energía mundial para el sector transporte, en gran medida responsable de grandes emisiones de dióxido de carbono $\left(\mathrm{CO}_{2}\right)$.

En México, la Secretaría de Medio Ambiente y Recursos Naturales (SEMARNAT 2017) menciona que los productos del petróleo son la principal fuente de energía para el sector transporte, debido a su alta densidad energética, por lo tanto, dicho sector es uno de los principales consumidores de combustible fósil. Por su parte, la Secretaría de Comunicaciones y Transportes (SCT 2017. Cuadro I), denomina al autotransporte federal de pasajeros, como el servicio destinado a la transportación de personas que se presta de manera regular en caminos federales, con itinerarios y rutas fijas, sujeto a horarios para la llegada y salida de vehículos en lugares autorizados para el ascenso y descenso de pasajeros en terminales registradas por la Secretaría.

Según la Comisión Nacional para el Uso Eficiente de la Energía (CONUEE 2017) el sector transporte es el mayor consumidor nacional de energía, con aproximadamente el $46 \%$ del consumo energético total de la última década, por lo que se hace necesaria una transición energética para que este tipo de transporte disminuya el consumo de energía proveniente del petróleo con el fin de mitigar el daño ambiental.

El objetivo de la presente investigación fue dar a conocer las preferencias de las empresas de autotransporte federal de pasajeros sobre los diferentes tipos de energías alternativas, ya que hasta hoy no han dejado de depender de la energía fósil, principalmente el diésel. Se utilizó la escala de Likert para analizar las respuestas de los encuestados, ya que dicha escala nos permite medir actitudes y conocer el grado de conformidad del encuestado con cualquier 
CUADRO I. ESTRUCTURA EMPRESARIAL DEL TRANSPORTE TERRESTRE DE PASAJEROS, EXCEPTO POR FERROCARRIL.

\begin{tabular}{lccccc}
\hline $\begin{array}{l}\text { Tipo de } \\
\text { empresa }\end{array}$ & $\begin{array}{c}\text { Estrato en } \\
\text { unidades }\end{array}$ & $\begin{array}{c}\text { Número de } \\
\text { empresas }\end{array}$ & $\%$ & $\begin{array}{c}\text { Número de } \\
\text { vehículos }\end{array}$ & $\%$ \\
\hline Hombre camión & 1 a 5 & 2177 & 72.5 & 3526 & 6.5 \\
Pequeña & 6 a 30 & 486 & 16.2 & 6933 & 12.7 \\
Mediana & 31 a 100 & 219 & 7.3 & 11855 & 21.7 \\
Grande & más de 100 & 119 & 4.0 & 32217 & 59.1 \\
Total & $*$ & 3.001 & 100 & 54531 & 100 \\
\hline
\end{tabular}

Fuente: Subsecretaría de Transporte, Dirección General de Autotransporte Federal. Estadística Básica del Autotransporte Federal (SCT 2017).

afirmación que se le plantee. Resulta especialmente útil emplearla en situaciones en las que se quiere que la persona matice su opinión (Llauradó 2014). A través de las diferentes respuestas de este sector se podrá identificar qué tipo de estrategias de sostenibilidad podrían usar.

\section{MATERIALES Y MÉTODOS}

Para esta investigación se consideraron cuatro tipos de estructura empresarial: hombre camión, empresa pequeña, empresa mediana y empresa grande.

La investigación fue de tipo observacional transversal. Se utilizó el programa STATS versión 2.0 para determinar el tamaño de la muestra de acuerdo con las siguientes características: a) el tamaño del universo (3001), b) el error estándar máximo aceptable (5\%), c) el porcentaje estimado de la muestra $(50 \%)$, probabilidad de ocurrencia de un fenómeno: $(\mathrm{p}+\mathrm{q}=1)$, p y q, serán del $50 \%$ cada uno, d) nivel deseado de confianza $(95 \%)$. El resultado arrojó un total de 341 empresas entrevistar.

El instrumento utilizado fue una encuesta en escala Likert. Dicha escala está formada por un conjunto de preguntas referentes a actitudes, cada una de ellas de igual valor. Los sujetos responden indicando acuerdo o desacuerdo. Se establecen generalmente cinco rangos (García et al. 2011). Los cinco rangos, con los valores de respuesta de actitud que se utilizaron en la presente investigación fueron: 5) totalmente de acuerdo, 4) de acuerdo, 3) ni de acuerdo ni desacuerdo, 2) en desacuerdo, 1) totalmente en desacuerdo; con 13 ítems a 341 empresas (unidad de análisis), de las cuales no contestaron tres, por lo cual únicamente se trabajó con 338 empresas. En la encuesta se realizaron preguntas cerradas referentes a la estructura empresarial.

Posteriormente se conjuntaron opiniones sobre las diversas preguntas que en resumen fueron: energía fósil (diésel), monitoreo de $\mathrm{CO}_{2}$, inversión y migración a tecnologías verdes, responsabilidad social, mentalidad sobre el ambiente, biodiésel, energía solar, energía eólica, energía híbrida o totalmente eléctrica, gas natural, hidrógeno, ley sostenible sobre el autotransporte de pasajeros y tendencias internacionales del sector. El resultado fue el porcentaje de cada uno de los valores de las cinco categorías Likert de acuerdo con la frecuencia de las respuestas; la medición de los datos se realizó con el programa Microsoft Office Excel, Windows 7, a través de la siguiente fórmula:

$f \mathrm{x}=\mathrm{B} 2+\mathrm{D} 2+\mathrm{F} 2+\mathrm{H} 2+\mathrm{J} 2$

En donde: $f \mathrm{x}=$ variables, $\mathrm{B} 2=$ totalmente de acuerdo, D2 = de acuerdo, F2 = ni de acuerdo, ni desacuerdo, H2 = En desacuerdo, J2 = Totalmente en desacuerdo.

\section{RESULTADOS Y DISCUSIÓN}

Con base en la información obtenida, conforme a las respuestas afirmativas de los empresarios, de acuerdo y totalmente de acuerdo, se da paso a los resultados. En las figuras se ilustra el porcentaje obtenido por cada valor de respuesta.

En la figura 1, se muestran resultados correspondientes al uso de energía fósil. Se puede observar que la mayoría de los empresarios están a favor de seguir utilizando el diésel con un $99.1 \%$. Cabe mencionar que cuando se realizaron las entrevistas, algunos de estos empresarios mostraron interés en el diésel de ultra bajo azufre (UBA). Actualmente en México los motores que usan diésel se basan en los certificados de la Agencia de Protección del Ambiente de los EUA (EPA, por sus siglas en inglés), norma 10 y la norma ambiental europea (EURO VI). Ambas 


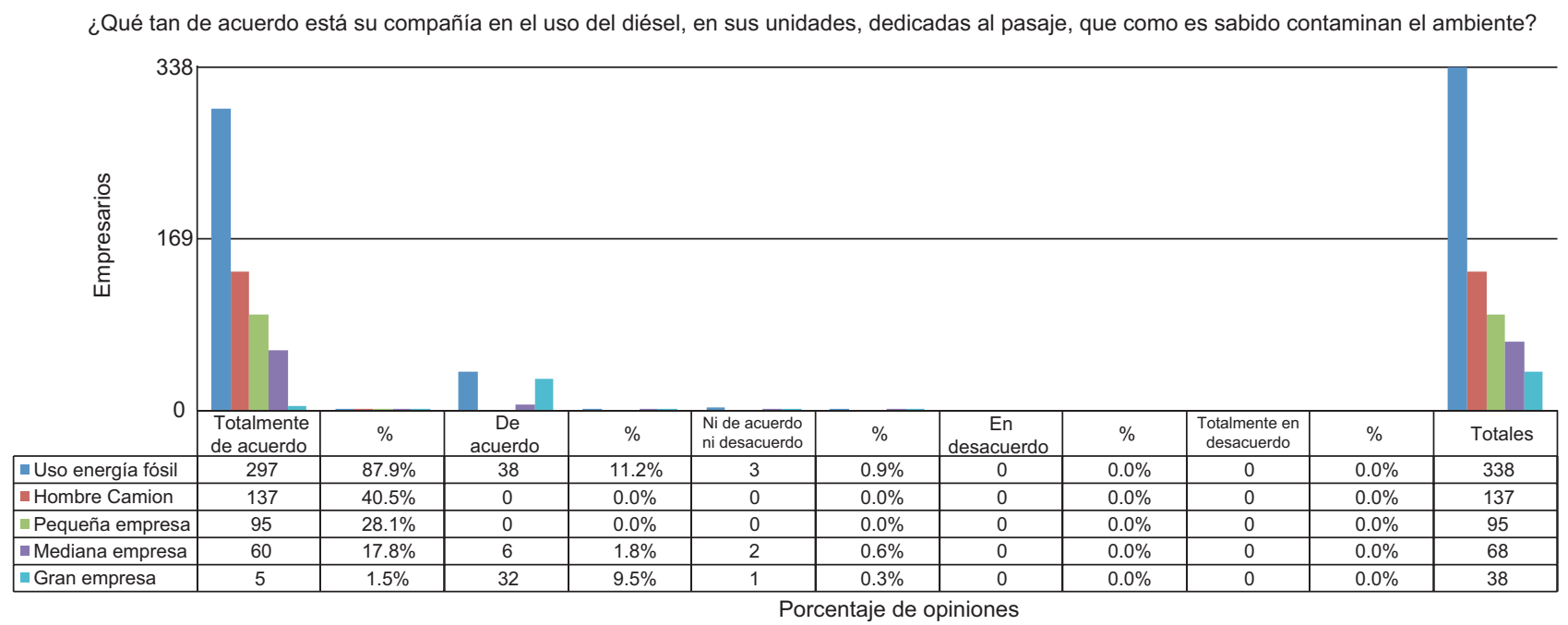

Fig. 1. Uso energía fósil (diésel).

certificaciones son indispensables para este tipo de vehículos, principalmente porque ayudan a que contaminen menos. Ferrari (2013) hace mención que tanto el cambio climático como los límites físicos a la producción de energías fósiles imponen cambios al paradigma económico dominante. Estamos viviendo un momento crucial de la historia de la civilización, donde las políticas energéticas y ambientales que se establezcan en el mundo en los próximos años determinarán si podremos transitar hacia una economía que pueda sustentarse con las energías renovables o dirigirnos hacia un futuro de conflictos por los recursos remanentes.
En la figura 2, se observa que el $69 \%$ de los empresarios están a favor de contar con un sistema de monitoreo de $\mathrm{CO}_{2}$, aunque algunos manifestaron su preocupación por la falta de recursos económicos, ya que sus unidades no son nuevas y creen que esto puede ser negativo para su compañía. Proponen que se les otorgue apoyo económico gubernamental para poder cumplir con este objetivo y llegar a realizarlo con la mejor eficiencia operativa, tecnológica y de bajas emisiones. Sin embargo, como menciona Núñez (2015) el sistema por el que las empresas obtendrían beneficios al intentar reducir las emisiones nunca ha llegado a tomar forma, el gobierno aún no ha

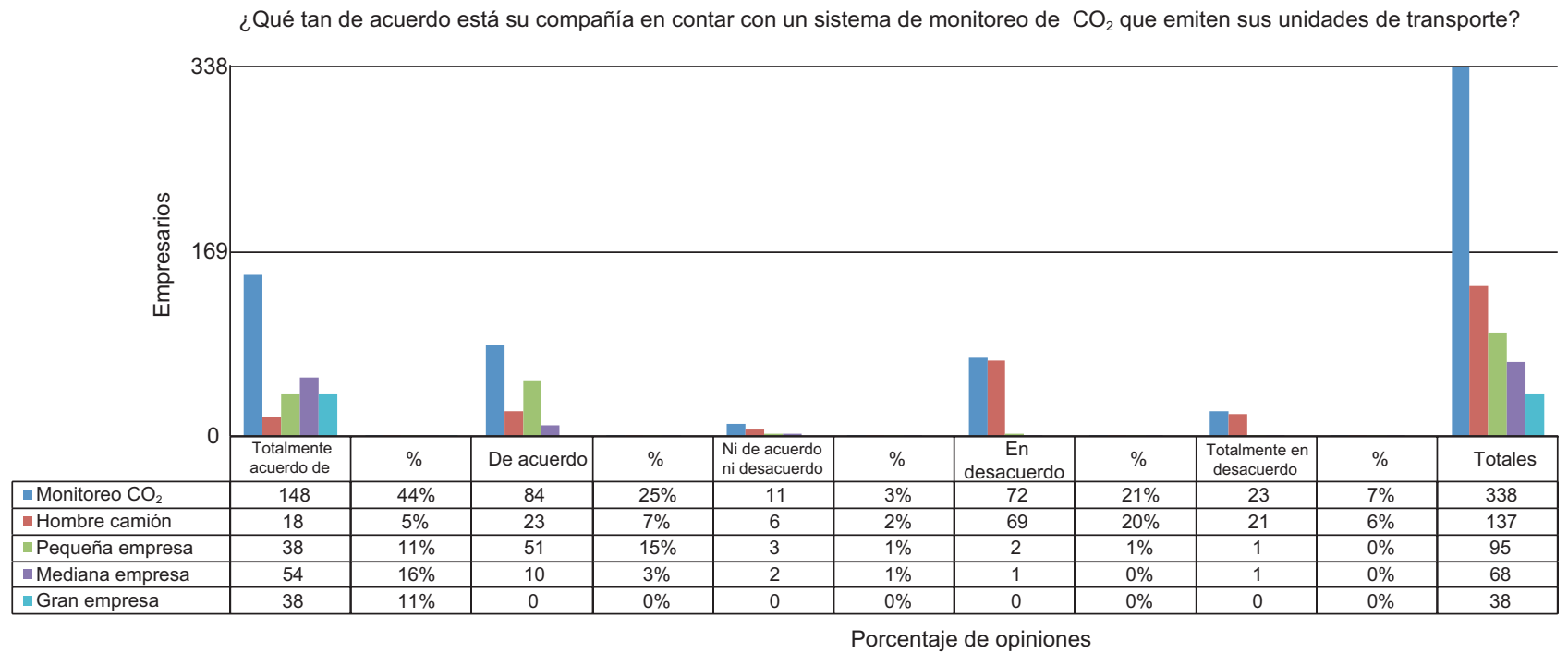

Fig. 2. Monitoreo $\mathrm{CO}_{2}$. 
estudiado esta posibilidad de apoyo hacia sistemas de monitoreo que deberían ser parte integral de estas unidades de transporte.

El $99 \%$ de los empresarios (Fig. 3) están a favor de invertir en innovación, investigación y desarrollo para la migración a tecnologías verdes o sostenibles. Las categorías hombre camión y empresa pequeña manifestaron no tener recursos suficientes para ello. La Comisión Económica para América Latina y el Caribe (CEPAL 2016) menciona que hay empresas con departamentos exclusivos para la innovación tecnológica, con grandes inversiones en investigación y desarrollo $(\mathrm{I}+\mathrm{D})$ y que en las pequeñas empresas el impacto ambiental debe incorporarse al proceso general de mejora de la productividad y la competitividad.

Como se puede observar en la figura 4, el $89 \%$ de los empresarios están a favor de implantar estrategias para que sus compañías sean consideradas socialmente responsables y minimizar daños al ambiente. Manifestaron que mejorarán en la medida de lo posible sus chasises y carrocerías, para asegurar la vida útil de sus vehículos en beneficio de sus clientes; además de generar un impacto social positivo a través de la cooperación entre los transportistas, gobierno y ciudadanía para mejorar su contexto. Es importante, como mencionan Chirinos et al. (2013), que las

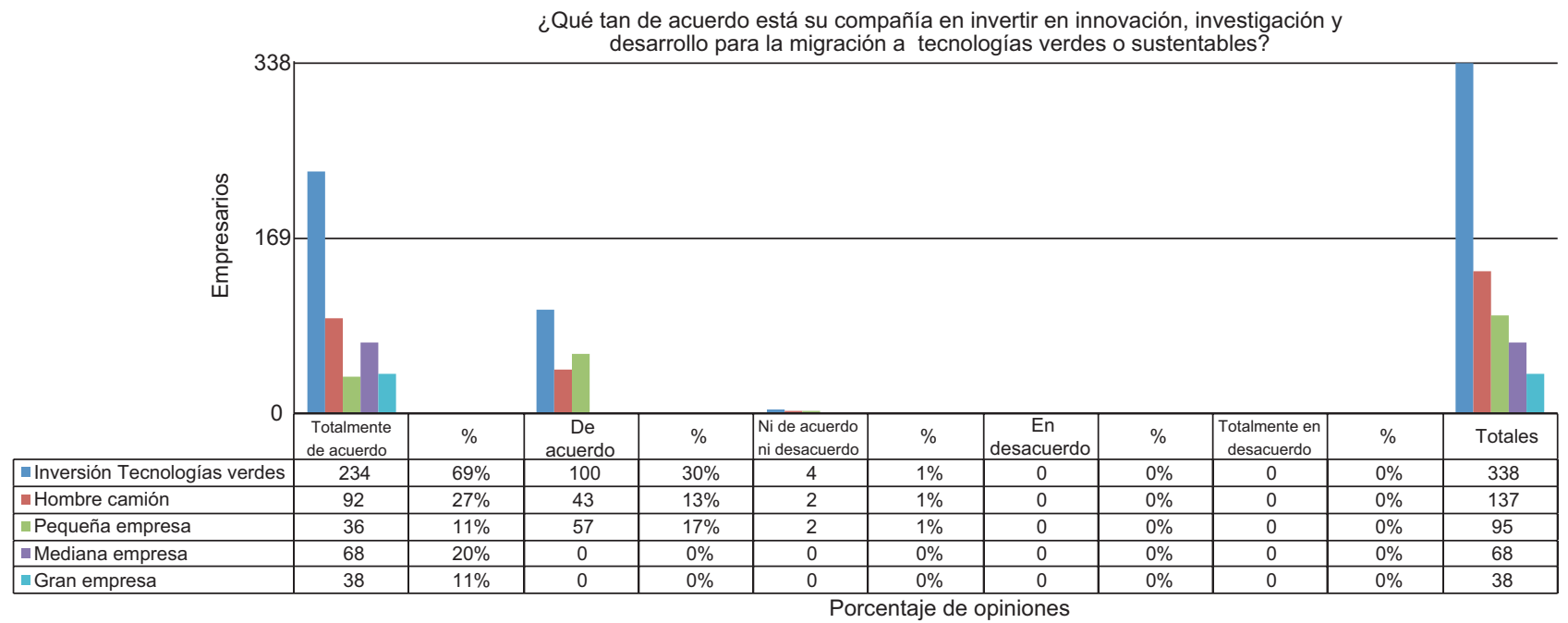

Fig. 3. Inversión y migración a tecnologías verdes.

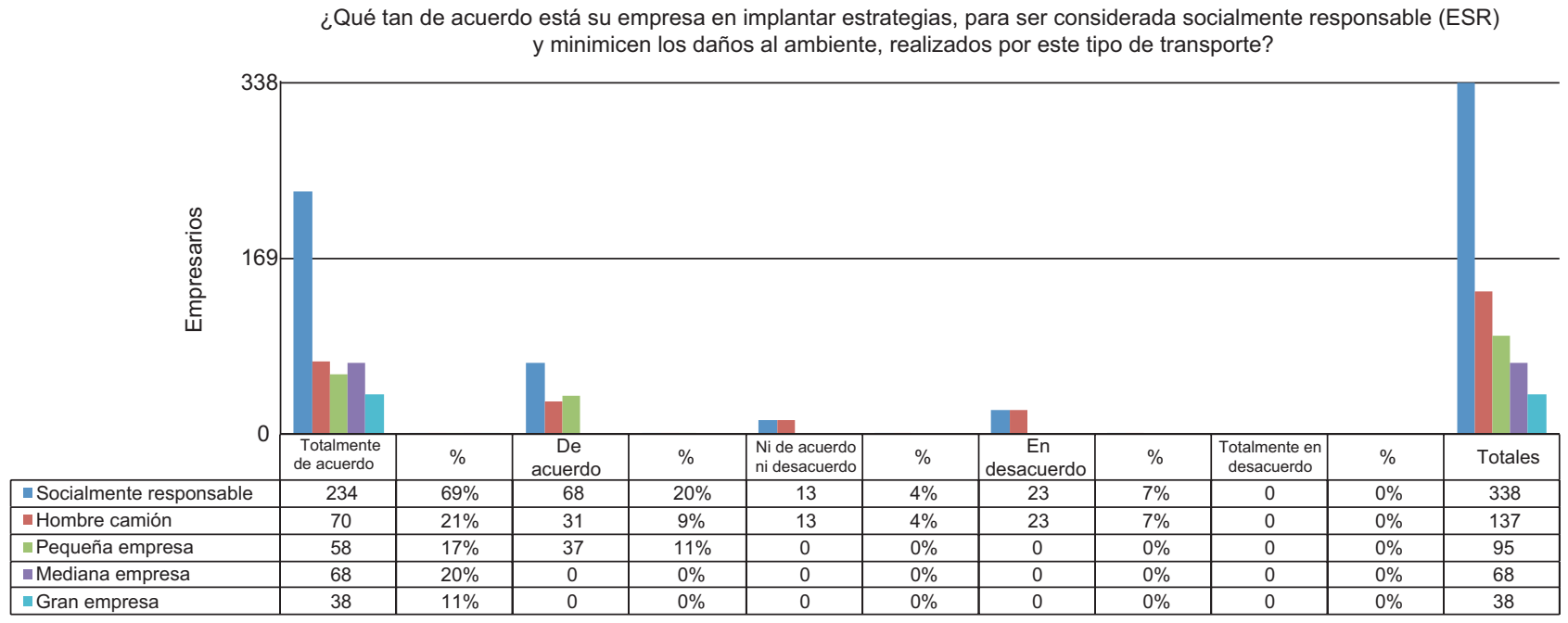

Porcentaje de opiniones

Fig. 4. Socialmente responsable. 
empresas socialmente responsables sean organizaciones inteligentes que comprendan el valor del bien común y no conciban otra forma de desarrollo que no sea la sostenibilidad; la ética debe ser la guía para hacer las cosas bien, pero hay que ir más allá, para retribuir al entorno y a la sociedad los beneficios obtenidos.

El $100 \%$ de los empresarios están a favor de cambiar su mentalidad hacia la sostenibilidad y el cuidado del ambiente (Fig. 5) y está preocupado por ello, sin embargo consideran a la crisis económica actual como el principal impedimento para las inversiones; aunque aseguraron que buscarán la forma para que desde sus diversas operaciones poco a poco vayan cambiando hacia buenas prácticas sostenibles y logren alinear sus objetivos, misión y visión independientemente del combustible que usan sus unidades. Estiman, como mencionan Roa y Pescador (2016), que es necesario plantear medidas para proteger la salud y promover la toma de conciencia, sensibilizándose ante los eventos de alteración de los factores ambientales. Asimismo, que también es necesario proponer investigaciones científicas que evalúen riesgos potenciales con el fin de planificar estrategias de adaptación y mitigación. En definitiva, se comprometieron a evolucionar desde el transporte urbano y foráneo a mediano y largo plazos y esperan pronto el uso de combustibles alternativos y energías limpias.

En la figura 6, se observa que el $100 \%$ de los empresarios está a favor del biodiésel, siempre y cuando cuenten con subsidio gubernamental, ya

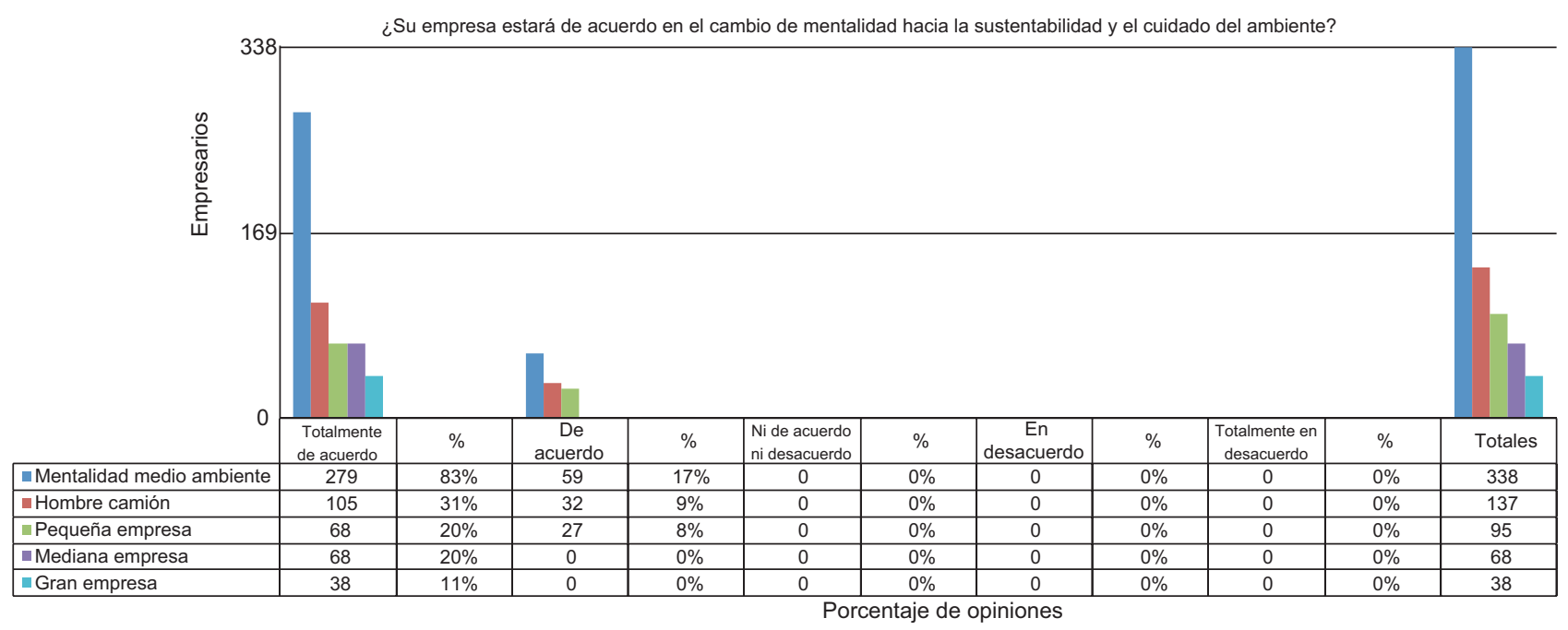

Fig. 5. Mentalidad sobre el ambiente.

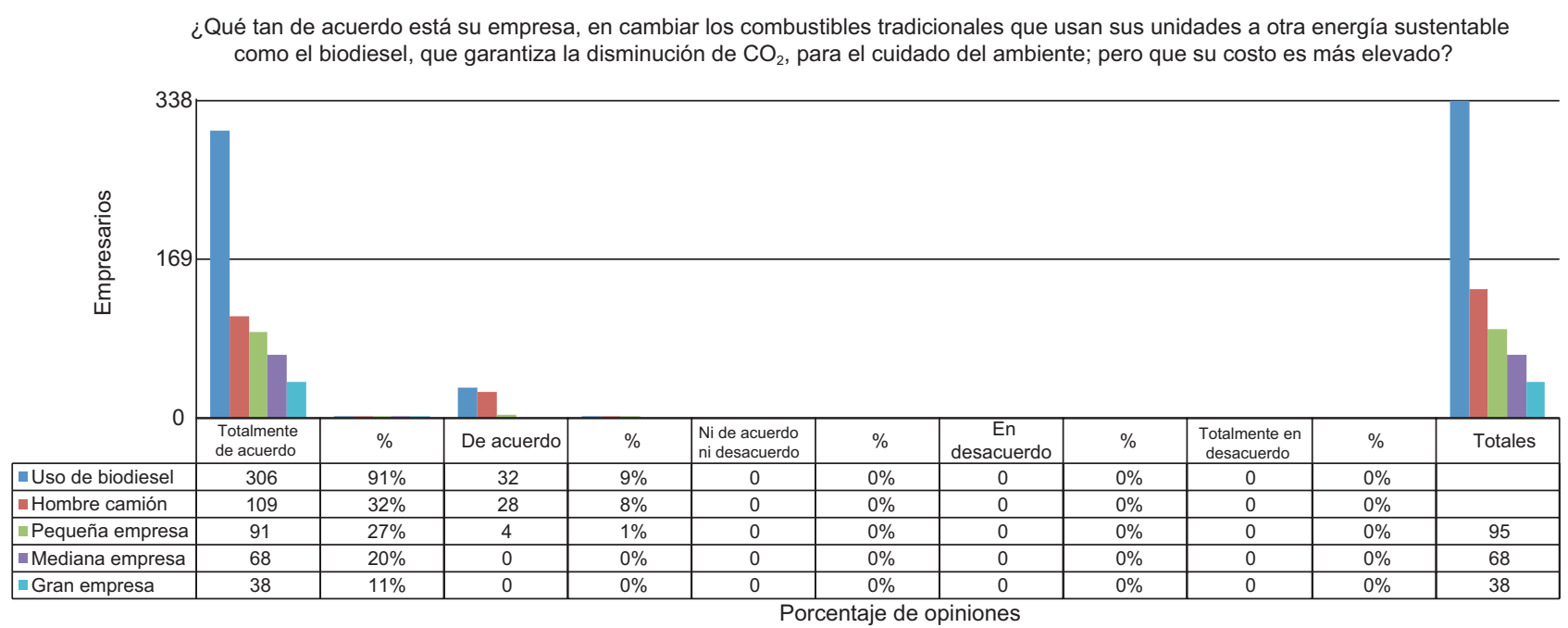

Fig. 6. Uso de biodiésel. 
que con este combustible ayudarían a disminuir la contaminación. El biodiésel, como menciona Castro (2012), tiene el potencial para reducir las emisiones de gases contaminantes generados en el sector transporte. Algunos empresarios comentaron que en Chiapas, Veracruz y Puebla, se hizo un intento por utilizar el biodiésel en camiones urbanos pero lamentablemente fracasó debido al poco apoyo de los gobiernos estatales, municipales y federal, a causa de su elevado gasto en infraestructura. Lo anterior a pesar de que la Secretaría de Energía (SENER) y la Secretaría de Agricultura, Ganadería, Desarrollo Rural, Pesca y Alimentación (SAGARPA), cuentan con estudios para que los empresarios que deseen invertir en alguna planta de biodiésel de mediana y gran escala lo hagan. Las grandes empresas, están en acuerdo en que instituciones como la Universidad Nacional Autónoma de México (UNAM), el Instituto Politécnico Nacional (IPN) y la Universidad Autónoma Metropolitana (UAM), desarrollen proyectos de este tipo de energía. Al respecto, Castro (2012) señala que en el mundo han sido identificadas alrededor de trescientos cincuenta plantas oleaginosas que pueden ser candidatas a investigación para la posible producción de biodiésel y su comercialización a bajo costo a escala industrial.

En la figura 7, se muestra que el $100 \%$ de los empresarios estuvo de acuerdo con el uso de la energía solar; en México la radiación solar es una fuente inagotable que pude ser aprovechada. Como menciona Trejo (2011) ésta aporta el mayor flujo de energía en los ecosistemas terrestres; se calcula que alrededor de 100000 tera watts $(1 \mathrm{TW}=1012$ watts) inciden sobre la superficie del planeta al año. De tener la capacidad para aprovechar la energía solar que se recibe en un año, considerando que la demanda energética global en ese mismo lapso es de alrededor de $16 \mathrm{TW}$, esta aseguraría el contar con más de 6000 veces los requerimientos actuales de energía de nuestra civilización. Hay países donde ya se está aprovechando este tipo de energía en el autotransporte, como en Adelaida, Australia, que es el primer país del mundo en poner en sus calles autobuses con energía solar, montando en el techo placas fotovoltaicas subvencionadas por el estado. Con una fuerte radicación solar, las placas producen el $70 \%$ de la energía necesaria para el funcionamiento de los autobuses (Adelaida Buscompany 2019) el otro 30\% proviene del sistema de frenado (EcoInventos 2016). Este ejemplo, podría ser un prototipo mundial para ser duplicado en México.

En la figura 8, se ve que el $98 \%$ de los empresarios está a favor del uso de la energía eólica; anuque les interesa ver un prototipo primero, verificar su funcionamiento y creen que sería una oportunidad hacia la sostenibilidad. En este aspecto México propone utilizar la energía eólica para cumplir con la meta del $35 \%$ de participación de energías limpias en la generación de energía eléctrica para el año 2024. Este tipo de energía tiene un papel importante en dicho cometido por sus beneficios ambientales, sociales y económicos. Además, su desarrollo tecnológico ayudará a la reducción de emisiones de $\mathrm{CO}_{2}$ (SENER 2016).

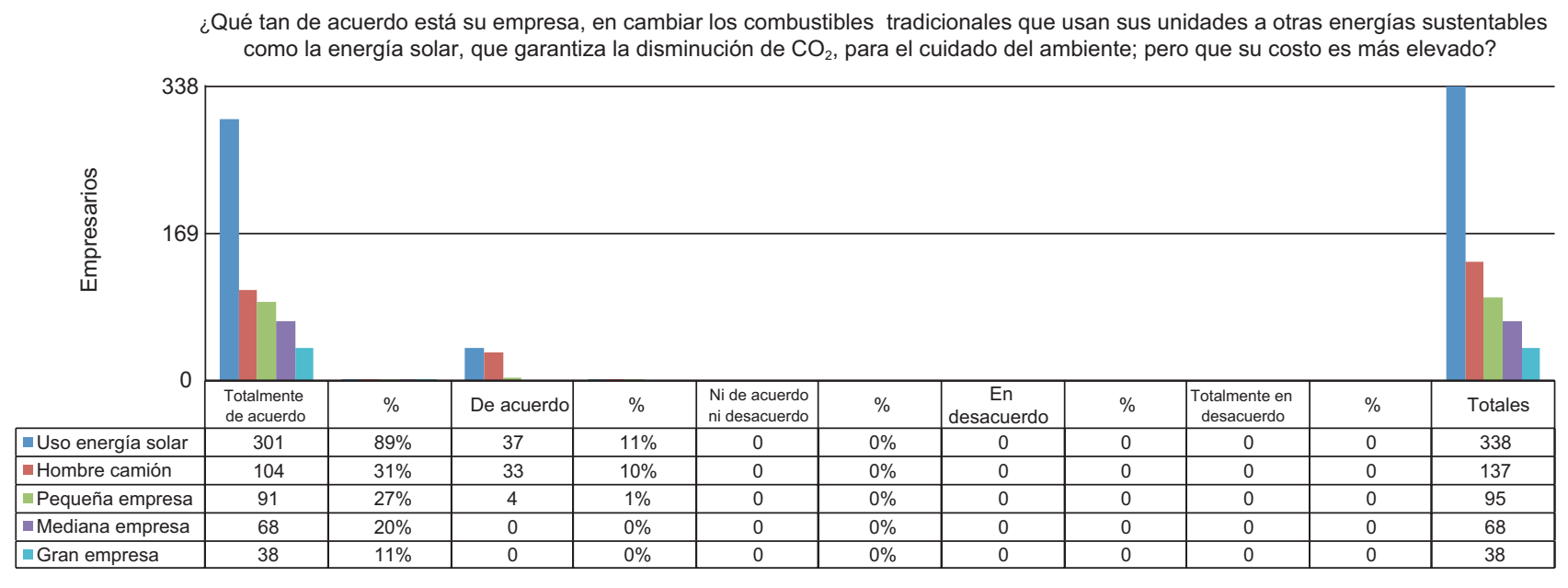

Porcentaje de opiniones

Fig. 7. Uso energía solar. 


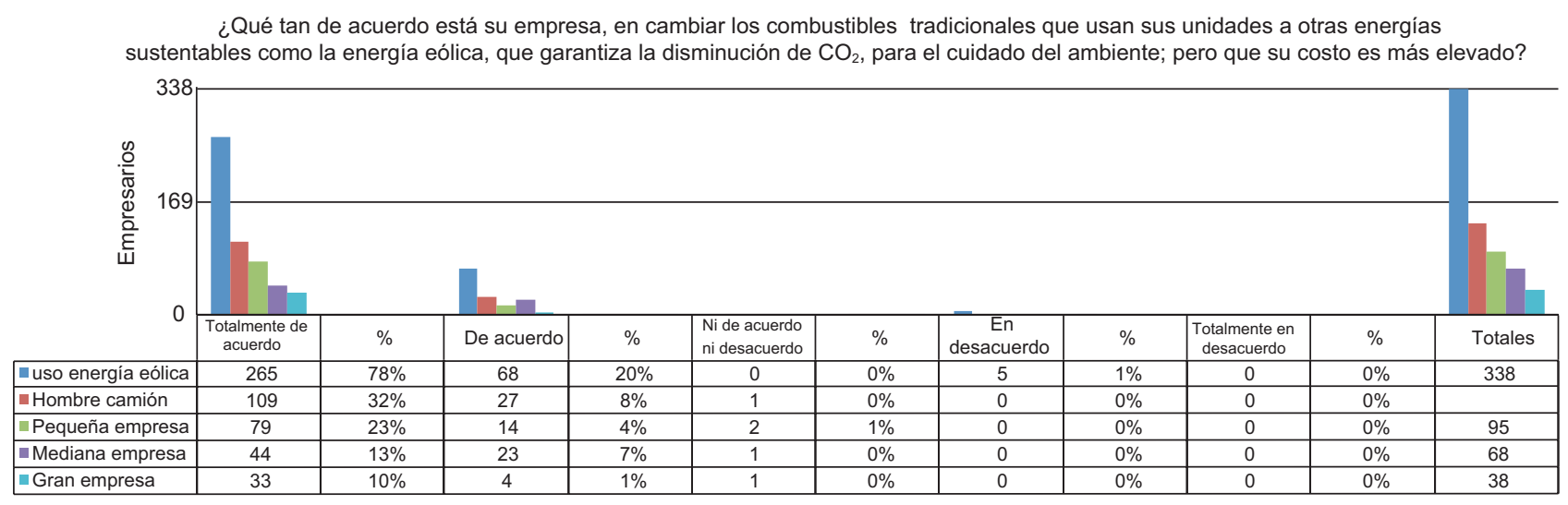

Porcentaje de opiniones

Fig. 8. Uso energía eólica.

En la figura 9, es evidente que al $100 \%$ de los empresarios le gustaría usar la energía híbrida (eléctrica-diésel) o totalmente eléctrica; opinaron que estos camiones pronto serán adquiridos en México y serán los que sustituyan a los actuales; solicitan apoyo gubernamental para poder comprarlos. Se sabe que mediante esta tecnología se elimina gran parte la emisión de contaminantes atmosféricos; un ejemplo es la Ciudad de México, donde diariamente se realizan más de 6 millones de viajes en los 25000 autobuses de la red de transporte público. La renovación de la flota con alternativas de electromovilidad ofrece una oportunidad única para el ambiente, pues en un año un autobús híbrido disminuye la emisión de 30 toneladas métricas de $\mathrm{CO}_{2}$, mientras que uno eléctrico usando energía renovable puede reducir hasta 90 toneladas. En este sentido, si bien se prevé que para 2030 los viajes en autobús aumenten cerca de
$50 \%$ y con esto se reduzca el uso de autos privados en las ciudades, lo cierto es que ese hecho por sí solo no garantiza la disminución de emisiones, pues los autobuses viajan hasta 10 veces más lejos que otros vehículos de pasajeros, generando mayor impacto ambiental especialmente cuando no tienen buen mantenimiento o necesitan ser renovados (García 2016)

En la figura 10, se muestra que el $100 \%$ de los empresarios están a favor de que sus flotas utilicen gas natural; saben que su costo es más económico que el del diésel y que las emisiones disminuyen considerablemente; piensan que es lo más inmediato que debe usarse a favor de la sostenibilidad, ya que los costos para convertir sus unidades no son tan elevados. Cabe mencionar que algunos de estos empresarios ya han convertido sus camiones, aún a sabiendas de que es un combustible fósil.. Existen compañías armadoras que ya desarrollan unidades

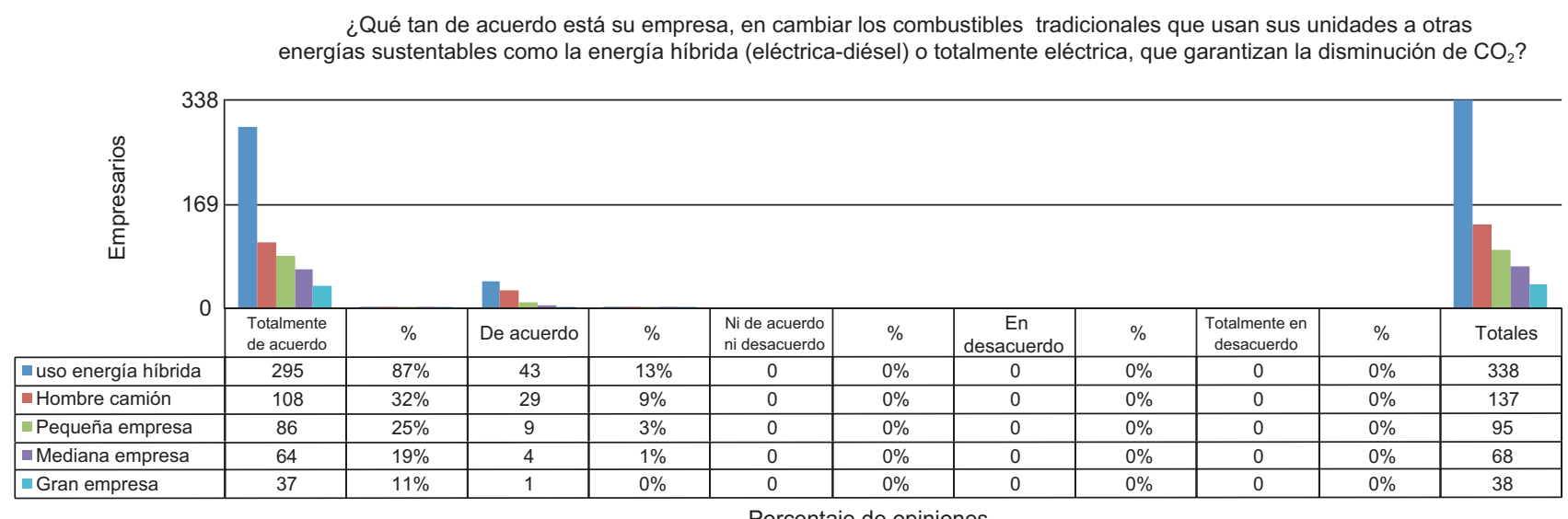

Porcentaje de opiniones

Fig. 9. Uso energía híbrida o totalmente eléctrica. 


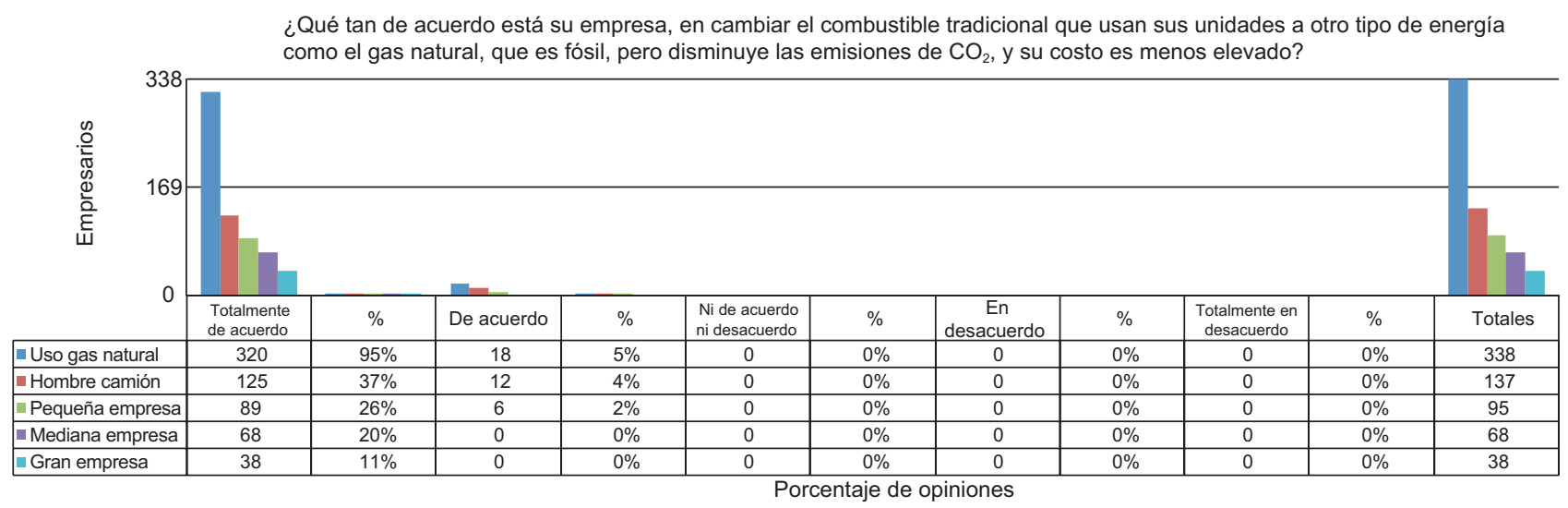

Fig. 10. Uso gas natural.

con este tipo de energía, lo que justifican explicando que su composición molecular es más simple que la de una molécula de diésel; por esto es más fácil su combustión y se generan menos emisiones contaminantes. Al comparar un motor diésel (EPA 2004) con uno de gas natural comprimido (EPA 2010) se observa que con el gas natural se reduce en más del $95 \%$ la emisión de partículas y en más del $90 \%$ la de óxidos de nitrógeno (NOx). Asimismo, también se reduce el ruido: 10 motores de gas natural comprimido producen el ruido equivalente de un motor de diésel. En cuanto a la viabilidad económica, aún sin los subsidios gubernamentales como los que tienen el diésel y la gasolina, el ahorro en el costo del gas natural, comparado con el diésel convencional, puede ser alrededor del $35 \%$. En seguridad, el gas natural no es tóxico y es más ligero que el gas LP, se disipa rápidamente en caso de fuga; los cilindros, válvulas y tubería que se instalan en el autobús cumplen con normas internacionales de seguridad. Actualmente el gas natural es considerado como la fuente de energía más limpia y respetuosa con el ambiente por lo que ocupa el tercer lugar en el mundo entre las fuentes de energía primaria más utilizadas después del petróleo y el carbón (Acevedo et al. 2013).

En la figura 11, se observa que el $100 \%$ de los empresarios favorecería el uso del hidrógeno; les interesa que ese tipo unidades se construya en México, sin embargo, a corto y mediano plazos ven difícil su uso en el transporte. Quintero y Nova (2013) mencionan que este tipo de energía representa grandes ventajas desde la perspectiva ambiental, la más importante es el hecho de que la combustión del hidrógeno no contamina. Existen dos razones principales por las que es deseable sustituir los combustibles fósiles por el hidrógeno: 1) la combustión del hidrógeno sólo produce como subproducto agua, mientras que los combustibles fósiles liberan $\mathrm{CO}_{2}$ que se fija en la atmósfera y es uno de los mayores responsables de lo que se denomina "efecto invernadero" y 2) las reservas de combustibles fósiles se agotarán tarde o temprano, mientras que el hidrógeno permanecerá inagotable.

De acuerdo con lo que se muestra en la figura 12, el $82 \%$ de los empresarios respondieron a favor de establecer la ley sobre autotransporte federal de pasajeros sostenible. Mencionaron que de alguna manera ya existe, al estar obligados los fabricantes de motores a cumplir con las normas del ambiente (EPA y EURO vigentes) sobre óxidos de nitrógeno (NOx), y partículas (PM), además de la Norma Oficial Mexicana NOM-167-SEMARNAT-2017 que establece los límites máximos permisibles de emisión de contaminantes para los vehículos automotores que circulan en entidades federativas como Ciudad de México, Hidalgo, Estado de México, Morelos, Puebla y Tlaxcala, así como los métodos de prueba para la evaluación de dichos límites y las especificaciones de tecnologías de información y hologramas (DOF 2012). Otros empresarios manifestaron que es acertado el establecimiento de esta ley, que marcaría el principio hacia la sostenibilidad forzosa del ramo y con ello habría mayores oportunidades de apoyo gubernamental, y un fortalecimiento regulatorio. Consideraron que se tendría que ayudar a las dos figuras empresariales más vulnerables (hombre camión y pequeña empresa). La promulgación de nuevas normas para vehículos más eficientes y el uso eficaz de la energía ayudará al país a cumplir su compromiso climático (Núñez et al. 2015).

Como se ve en la figura 13, el $100 \%$ de los empresarios está a favor de que las tendencias internacionales determinen el futuro del sector en México, pues es uno de los factores indispensables para la transferencia de 
¿Qué tan de acuerdo está su empresa, en cambiar los combustibles tradicionales que usan sus unidades a otras energías sustentables como el hidrógeno, que garantiza la disminución de $\mathrm{CO}_{2}$, para el cuidado del ambiente; pero que su costo es más elevado?

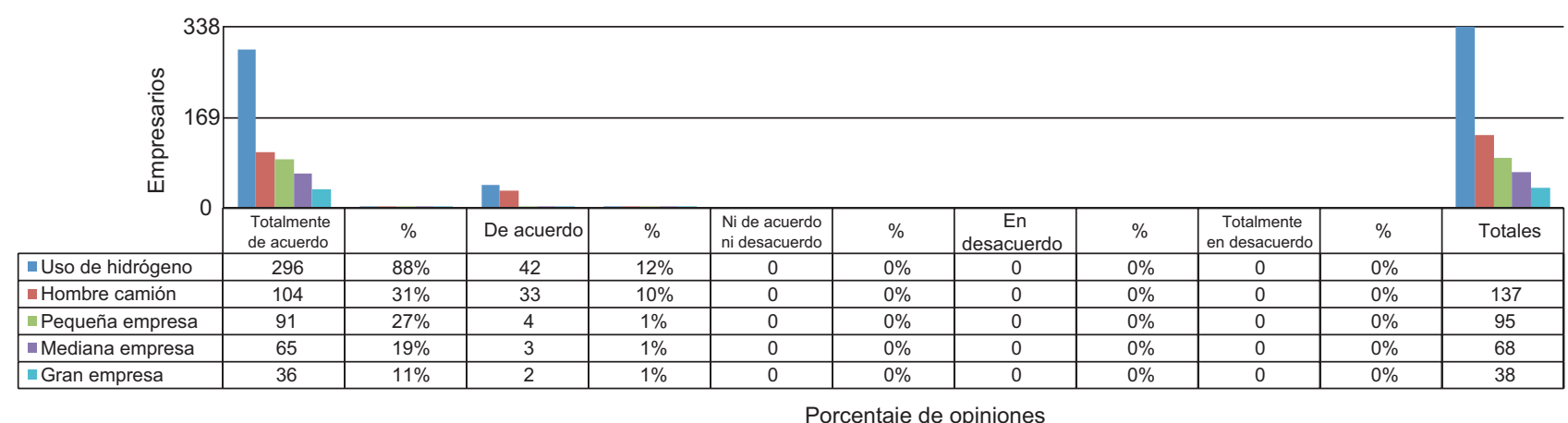

Fig. 11. Uso de hidrógeno.

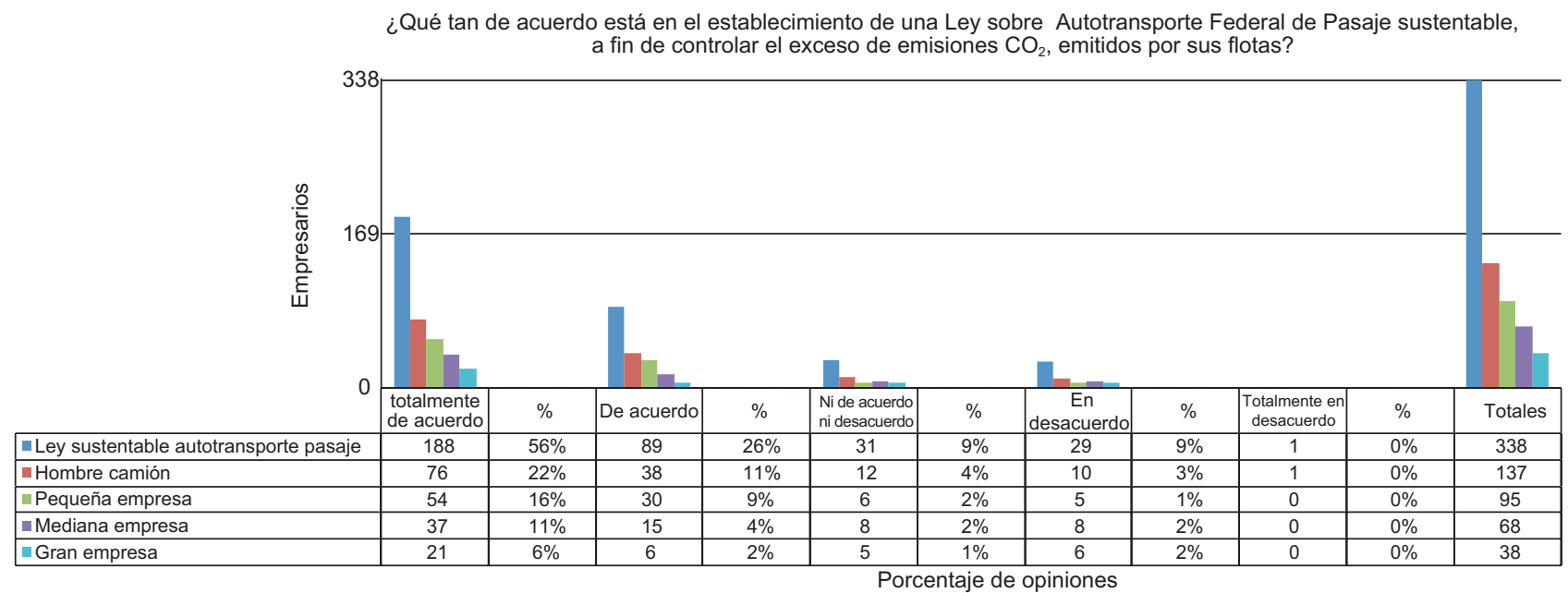

Fig. 12. Ley sostenible sobre el autotransporte de pasajeros.

tecnología. Ésta se ha logrado gracias a la globalización y han podido entrar más compañías armadoras con diferentes tipos de camiones, de los cuales ellos pueden elegir los que más les convenga. Un ejemplo es cómo el transporte y la circulación de vehículos en Norteamérica fueron gestados por largos intercambios comerciales y culturales entre las naciones de la región y que con el TLCAN se profundizaron (Camarena 2014). Sin embargo, el que México sea un país globalizado con 12 tratados de libre comercio con 46 países, puede ser un arma de dos filos: por un lado se tiene la oportunidad de compartir experiencias e intercambiar tecnologías con cualquiera de estos países en este ramo; pero por otro lado se corre el peligro de que México se convierta en un importador de tecnología y conocimiento, quedando en desventaja. No debemos olvidar que el fin primordial es encontrar mecanismos de mitigación o una estrategia sostenible que ayude a frenar el cambio climático y se lleve a cabo la Agenda 2030 para el Desarrollo Sostenible. Esto implica la consecución conjunta de diferentes objetivos, en particular, avanzar en el crecimiento económico y en el desarrollo social y ambiental, para lo cual es fundamental lograr el pleno empleo, promover la industrialización inclusiva y fomentar la innovación, considerando los efectos sobre el cambio climático y los impactos ambientales (CEPAL 2016). Lo anterior, especialmente en la industria del transporte, en la que en los actores del sector deberán buscar la estrategia sostenible, con resultados en beneficio del ambiente y a favor del uso de energías alternativas y limpias, diferentes a la fósil.

\section{CONCLUSIONES}

De acuerdo con las respuestas a las trece variables, se puede considerar que los empresarios del 


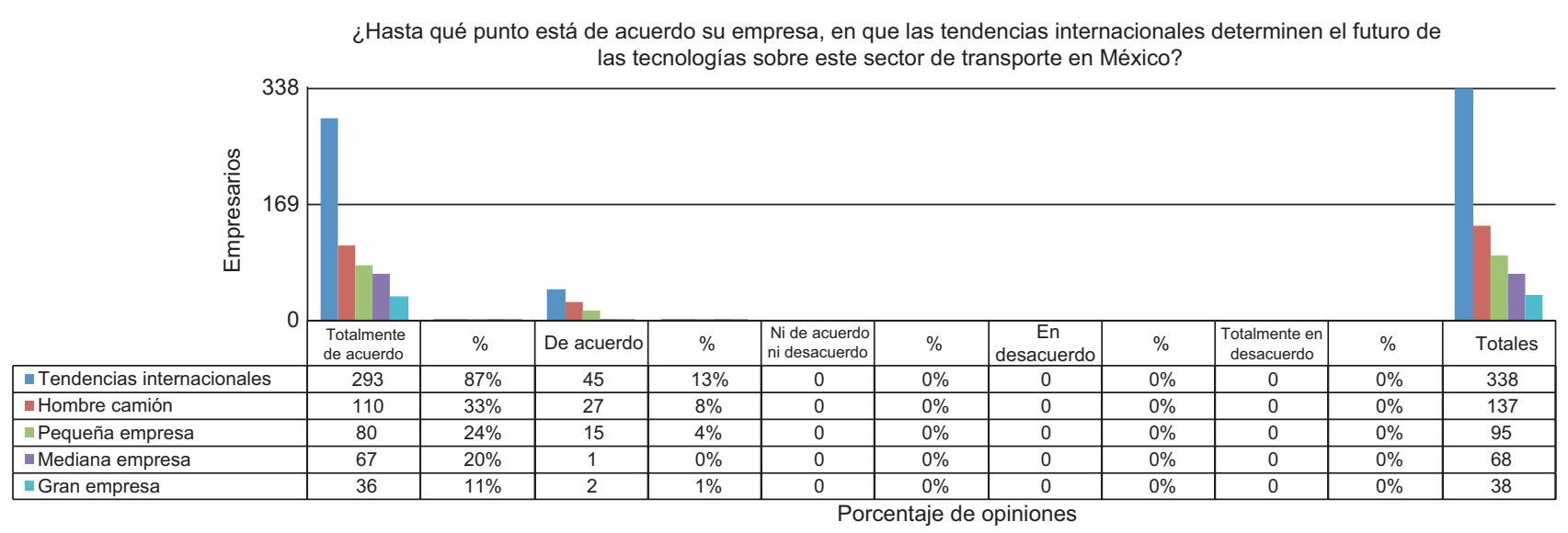

Fig. 13. Tendencias internacionales del sector.

autotransporte federal de pasajeros en México están dispuestos a adoptar una estrategia de sostenibilidad que disminuya el daño al ambiente causado por el uso de energía fósil. Los empresarios creen que la respuesta a corto y mediano plazos dependerá de factores económicos, sociales, gubernamentales y empresariales. Asimismo, que el apoyo gubernamental y de la Cámara Nacional del Autotransporte de Pasaje y Turismo (CANAPAT) que los representa podría tener un gran significado en el proceso de mitigación. En especial para las empresas hombre camión y pequeña empresa que necesitan particular atención en el factor económico, por ser las figuras empresariales más vulnerables del sector. En términos generales, para la migración hacia las energías limpias de bajas emisiones, pero sobre todo con beneficios a la sociedad en transporte rápido, seguro, y con la mayor tecnología, los empresarios del ramo deben contar con el apoyo tecnológico necesario para que puedan ofrecer a sus clientes un mejor servicio. Es importante que sus unidades cuenten con los más estrictos controles de calidad tanto en los materiales como en los procesos de producción por parte de las compañías armadoras. Los empresarios del sector deben establecer planes de negocio disruptivos y coordinados con los tres niveles de gobierno y con las instituciones académicas de educación superior, tanto públicas como privadas, con prospectiva a mediano y largo plazos. Quedan varios retos por resolver: legislación y apoyos gubernamentales significativos para la migración tecnológica así como la cooperación y experiencia internacional que permitan a los empresarios del autotransporte de pasajeros en México, ir hacia una evolución en el rubro, con el objetivo primordial de establecer una estrategia energética sostenible baja en $\mathrm{CO}_{2}$, con un impacto positivo para el ambiente y la conservación de la vida en el planeta.

\section{AGRADECIMIENTOS}

Agradezco al apoyo para llevar a cabo este proyecto a la Universidad Anáhuac México, a la Secretaría de Energía a través de la Comisión Nacional para el Uso Eficiente de la Energía (CONUEE), y a las empresas Dina de México y Geonúcleo de Servicios México.

\section{REFERENCIAS}

Acevedo O. L., Jaramillo J.E. y Blanco H.A. (2013). Estimación de emisiones de GEI (CO2 y CH4) generadas durante el transporte de gas natural en Colombia, aplicando metodología IPCC. Revista Fuentes 11 (2), 43-51.

Camarena L.M. (2014). Transporte terrestre, un tema regional estratégico del Tratado de Libre Comercio de América del Norte. Norteamérica 9 (2), 205-224.

Castro M.C., Beltrán L.I. y Ortiz J.C. (2012). Producción de biodiésel y bioetanol: ¿una alternativa sostenible a la crisis energética? Ra Ximhai 8 (3), 93-100.

CIIFEN (2018). El niño/la niña en américa latina. Centro Internacional para la Investigación del Fenómeno de El Niño [en línea]. http://www.ciifen.org/ 29/11/2017

CMMAD (1987). Nuestro futuro común. Comisión Mundial del Medio Ambiente y el Desarrollo. Alianza Editorial, Madrid, España, 460 pp.

CEPAL (2016). Ciencia, tecnología e innovación en la economía digital. La situación de américa latina 
y el caribe. Comisión Económica para América Latina y el Caribe. Naciones Unidas, Santiago de Chile, 29 pp.

CONUEE (2017). Hoja de ruta en materia de eficiencia energética. Comisión Nacional para el Uso Eficiente de la Energía [en línea]. https://www.gob.mx/conuee/ acciones-y-programas/hoja-de-ruta-de-eficienciaenergetica 25/11/2016

Corona M.A. (2018). El conocimiento, la percepción y disponibilidad para afrontar el cambio climático en una población emergente, los migrantes de retorno. Estudios Sociales. Revista de Alimentación Contemporánea y Desarrollo Regional 28 (52), 3.

Cham K.W. y Maubourne R. (2005). La estrategia del Oceano azul, Harvard Business School Press, México, $332 \mathrm{pp}$.

Chirinos M.E., Fernández L. y Sánchez G. (2013). Responsabilidad empresarial o empresas socialmente responsables. Razón y Palabra (81), 1-17.

DOF (2012). Ley general de cambio climático. Diario oficial de la federación. Documento oficial, Ciudad de México, México, 30 pp.

DGAF (2017). Estadística básica del autotransporte federal. Dirección General de Autotransporte Federal [en línea]. http://www.sct.gob.mx/transporte-y-medicinapreventiva/autotransporte-federal/estadistica/2017/ $8 / 04 / 2018$

EcoInvetos (2016). En Australia, autobús gratis gracias a la energía solar [en línea]. https://ecoinventos.com/ AUSTRALIA-AUTOBUS-GRATIS-GRACIAS-ALA-ENERGIA-SOLAR/ 2/03/2018

Ferrari L. (2013). Energías fósiles: diagnóstico, perspectivas e implicaciones económicas. Revista Mexicana de Física 59 (2), 36-43.

García A. (2016). Electromovilidad, clave para un transporte sostenible: volvo buses [en línea]. https://tyt. com.mx/noticias/electromovilidad-clave-para-untransporte-sostenible-volvo/ 13/01/2018

García J., Aguilera J. R. y Castillo A. (2011). Guía técnica para la construcción de escalas de actitud [en línea]. https://odiseo.com.mx/articulos/guiatecnica-para-la-construccion-de-escalas-de-actitud/ 20/02/2018

Lara C., Mendoza J.F., López M.G., Téllez R., Martínez W. y Alonso E.M. (2009). Propuesta metodológica para la estimación de emisiones vehiculares en ciudades de la república mexicana. Publicación Técnica $322,1-91$.
Minzberg H. y Quinn J.B. (1988). El proceso estratégico, conceptos, contextos y casos [en línea]. https://isabelportoperez.files.wordpress.com/2012/08/proceso_estrategico_mintzberg.pdf 13/03/2018

Miklos T. y Tello M.E. (1998). Planeación prospectiva, una estrategia para el diseño del futuro. Limusa, México, $200 \mathrm{pp}$.

Núñez C.A., González M.M. y Saura L.G. (2015). Proceso de recuperación de $\mathrm{CO} 2$. Generalidades ICIDCA sobre los derivados de la caña de azúcar. Instituto Cubano de Investigaciones 49 (2), 24-32.

Llauradó O. (2014). La escala de likert: qué es y cómo utilizarla [en línea]. https://www.netquest.com/blog/es/laescala-de-likert-que-es-y-como-utilizarla 23/09/2019

Porter E.M. (1999). Ser competitivo, nuevas aportaciones y conclusiones. Deusto Editorial, Madrid, España, 473 pp.

Quintero J.R. y Nova D.C. (2013). El hidrógeno como combustible alternativo en la producción de energía y su implementación en el transporte vehicular. L'esprit Ingénieux 4 (1), 114-125.

Roa L.A. y Pescador B. (2016). La salud del ser humano y su armonía con el ambiente. Universidad Militar Nueva Granada. Revista MED 24 (1), 111-122. https:// doi.org/10.18359/rmed.2338

SCT (2017). Estadística básica del autotransporte federal. Secretaría de Comunicaciones y Transportes [en línea]. http://www.sct.gob.mx/transporte-y-medicina-preventiva/autotransporte-federal/estadistica/ 23/01/2018

SEMARNAT (2017). Ciclo de vida para tecnologías de transporte bajas en carbono. Secretaría de Medio Ambiente y Recursos Naturales [en línea]. https:// cambioclimatico.gob.mx/analisis-de-ciclo-de-vidapara-tecnologias-de-transporte-seleccionadas-bajasen-carbono/ 23/01/2018

SENER (2016). Prospectivas del sector energético. Secretaría de Energía [en línea]. https://www.gob.mx/ sener/documentos/prospectivas-del-sector-energetico 25/08/2018

Thompson A.A., Peteraf M.A., Gamble J.E. y Strickland A.J. (2012). Administración estratégica. 18 ed. McGrawHill, México, 699 pp.

Trejo F. (2011). Energía solar: un futuro para México [en línea]. https:/expansion.mx/opinion/2011/07/13/ energia-solar-un-futuro-para-mexico 13/02/2018

Trentadue C. y Carranza H. (2014). La eficiencia energética en el transporte [en línea]. http://www.petrotecnia. com.ar/octubre2014/Pdfs_SIN_Public/LaEficiencia. pdf 20/05/2018 